\title{
Optical characterisation of polymeric nanocomposites using tomographic, spectroscopic and Fraunhofer wavefront assessment
}

\author{
Triantafillos Koukoulas*a, William R. Broughton ${ }^{\mathrm{a}}$, John Williams ${ }^{\mathrm{b}}$, Sameer Rahatekar ${ }^{\mathrm{b}}$ \\ ${ }^{a}$ National Physical Laboratory, Hampton Road, Teddington, Middlesex, TW11 0LW, UK; \\ ${ }^{\mathrm{b}}$ ACCIS, Department of Aerospace Engineering, University of Bristol, Bristol, BS8 1TR, UK
}

\begin{abstract}
Polymers are often embedded with specific nanofillers such that the functional characteristics and properties of the resulting polymeric nanocomposite (PNC) are enhanced. The degree to which these enhancements can be achieved depends not only on the level of particle loading of nanofillers, but most importantly on the resulting dispersion profile achieved within the matrix. Agglomeration (often referred to as clustering) is a result of the mixing process and very much depends on the chemistry between the polymer and nanofiller. Depending on the PNC type, different mixing processes can be applied but the general consensus is that such processes are not repeatable themselves. Not only it is quite difficult to achieve the desired level of dispersion, but in addition there is a limited number of characterization tools that can be employed to routinely check the homogeneity achieved within a produced sample. Transmission electron microscopy (TEM) and X-ray diffraction (XRD) techniques are usually employed, but they are very time consuming, expensive, require special sample preparation and treatment, often produce results that are difficult to interpret and can only analyse very small areas of sample. This work reports on the adaptation and development and three optical techniques that are non-destructive, can accurately characterize the dispersion achieved as a result of the mixing process and can analyse larger material areas. The techniques reported are based on static and dynamic visible and infra-red light scattering.
\end{abstract}

Keywords: polymeric nanocomposites, optical coherence tomography, wavefront correlation, photon correlation spectroscopy

*triantafillos.koukoulas@npl.co.uk; phone+44 (0)20 89436316

\section{INTRODUCTION}

The physical and mechanical, amongst other, properties of polymeric nanocomposites (PNCs), can be significantly enhanced by the inclusion of nanoparticles in small concentrations. Though such specific property enhancements can scale up according to the level of particle concentration present (wt. \%), an effect that can significantly reduce, or even forbid, such realization relates to particle agglomeration, also frequently referred to as dispersion. This quality relates to the level of homogeneity achieved within the polymeric matrix as part of the mixing process. Higher levels of dispersion clearly enable the specific material properties to be realised to the required degree, whereas poor dispersion levels result into localized clustering thus prohibiting such a functional performance and enhancement.

At present, dispersion is only referred to as a qualitative property rather than a quantitative one. As such, the meaning of "good", "moderate" or "poor", though being the current industrial and academic terminology, can be quite subjective. A considerate amount of research has been applied into different mixing processes to reduce the presence of agglomeration; however, for the vast majority of cases, this process is not quite repeatable at all.

The resulting PNC matrix can be assessed with a number of different techniques, all with their own merits and weaknesses. A most comprehensive review of the applied techniques ${ }^{1}$, includes scanning electron or probe microscopy (SEM and SPM respectively), atomic force microscopy (AFM), nanoindentation, infrared spectroscopy (IRS), dielectrics, electrical impedance tomography (EIT), Raman spectroscopy, ultrasonic resonance spectroscopy, scanning acoustic microscopy (SAM), laser ultrasound, spectro-photometry, all with very limited levels of success. Rheology can be applied with a certain degree of success to provide exfoliation and dispersion information but it is only applicable to

Optical Systems Design 2012, edited by Pablo Benítez, et al., Proc. of SPIE Vol. 8550, 855039 • @ 2012 SPIE CCC code: $0277-786 / 12 / \$ 18 \cdot$ doi: $10.1117 / 12.981182$

Please refer to any applicable publisher terms of use. 
polymeric melts ${ }^{2,3}$. X-ray diffraction (XRD) is a powerful technique that can be applied to the study of polymeric materials $^{4-6}$ providing information on the degree of exfoliation and intercalation of particulates within the sample. However, the interpretation can be difficult due to peak broadening resulting from the superposition of multiple peaks ${ }^{4}$. Transmission electron microscopy is also a powerful technique that can provide high quality visual representation of the polymeric matrix with an atomic resolution $(0.1 \mathrm{~nm})^{7,8}$ but special preparation of the samples is required (trimming) in order to enable the transition of the electron beam through the sample under investigation.

When combined, TEM and XRD can provide more accurate metrics and characterisation of PNCs than used on their own capacity ${ }^{9}$. However, the major drawback associated with these techniques is the long measurement and analysis times, in addition to the very small areas that can be studied at any one time ( $\mathrm{nm}$ to $\mu \mathrm{m}$ region). A common assumption lies on the fact that the characteristics and dispersion profile of the measurement area are applicable to the entire volume of the PNC, which can be in then $\mathrm{mm}$ to tens of $\mathrm{cm}$ region. The required equipment for such analysis is not suitable for installation for in-situ characterization; the common consensus has revealed that alternative techniques that can analyse larger sample areas in a fast and efficient manner, would facilitate the research currently been undertaken into the mixing processes required to achieve high levels of dispersion.

The experimental work presented, concentrates on three optical techniques that can provide dispersion information of large areas of such polymeric materials. Fourier domain optical coherence tomography in the infra-red, wavefront correlation and oscillatory photon correlation spectroscopy in the visible parts of the spectrum can be applied to PNC to reveal the level of homogeneity achieved after the mixing process. Most importantly, the techniques are applicable to transparent and semi-transparent solids, are non-destructive and non-evasive and can also discriminate between different levels of particle concentration.

\section{MATERIALS INVESTIGATED}

Material 1: Organoclay Nanomer ${ }^{\circledR}$ 1.30E, an octadeylamine modified montmorillonite, reinforced Huntsman LY 564 epoxy resin with Ardur 2954 hardener with varying particle loading and dispersion profiles. The materials, listed in Table 1, were supplied by BAe Systems (Operations) Ltd in the form of $3 \mathrm{~mm}$ thick autoclave cured plaques with varying degrees of particle loading and dispersion. The autoclave cure cycle consisted of an initial temperature ramp to $80{ }^{\circ} \mathrm{C}$ at $4{ }^{\circ} \mathrm{C} / \mathrm{min}$, dwell of 1 hour at $80^{\circ} \mathrm{C}$ followed by cooling to room temperature (15-18 hrs). Pressure was ramped to $\sim 80 \mathrm{psi}$ at $14 \mathrm{psi} / \mathrm{min}$ at the start of the cure cycle. The plaques were post-cured at $140{ }^{\circ} \mathrm{C}$ for $8 \mathrm{hrs}$. Average planar dimensions of the nanoclay particles (platelets) were $0.74 \pm 0.34 \mathrm{~mm}$ and $0.51 \pm 0.22 \mathrm{~mm}$, respectively. Intragallery $\left(\mathrm{d}_{001}\right)$ spacing is $1.8-2.2 \mathrm{~nm}$ (Nanocor technical datasheet for Nanomer ${ }^{\circledR} 1.30 \mathrm{E}$ ).

Material 2: matrix material was Araldite DBF with HY951 hardener, while the scatterer material was Silica monodisoperse microspheres from Whitehouse scientific, part no MSSL001, with a size distribution, mean diameter 1.6um+-0.3, all between 1 and 2um and mix ration of 10:1. The particles were weighted out using a microbalance and the DBF resin was added (similarly weighed out). The material was then thoroughly mixed using a dual-centrifugal mixer SpeedMixer DAC150 PVZ/K, with typical spin cycles of 3500rpm for several minutes at a time. To help break clumps the material was refrigerated before mixing and care was taken to allow the mixture to not warm up too much. Once no clumps were visible the hardener was weighed out and then mixed in with the centrifugal mixer as before, but only for one mix cycle. The mixed material was poured into small sample pots (approx. 12g) and then degassed for 3 minutes at a pressure below 50mbar (not sure exact value, pumping continuously). Very few small bubbles were visible during the degassing. Once degassed the pots were transferred to an oven for curing at around $60 \mathrm{degC}$ and left for 48 hours to ensure they were fully cured.

\section{OPTICAL COHERENCE TOMOGRAPHY}

Optical coherence tomography is a non-evasive technique based on low coherence interferometry applied mostly in medical applications in order to acquire depth visual representation. The measurement beam penetrates the specimen under investigation, while the variation in refractive index gives rise to an increase in backscattered photons ${ }^{10}$. These photon sequences are subsequently analysed such that visual information from adjacent layers within the sample (e.g. human tissue or eye) is acquired. There are different processing techniques in $\mathrm{OCT}^{11}$ applied to obtain depth-resolved 
information, but they can be broadly divided into time or Fourier domain techniques. Fourier based techniques have certain advantages over time domain techniques ${ }^{12}$ and in particular, swept mode Fourier analysis increases the measurements sensitivity and accuracy ${ }^{13}$.

For this experimental study, Fourier domain optical coherence tomography (FD-OCT) was used with a commercially available interferometer system, more specifically Michelson Diagnostics EX1301 OCT microscope. This system operates at a frequency swept mode with a centre wavelength of $1305 \mathrm{~nm}$ and a bandwidth of $150 \mathrm{~nm}$. The system consists of four laser beams that are focused at different depths (inside the materials under investigation) over a depth of focus of $0.25 \mathrm{~mm}$ each, thus providing a total focal range of $1 \mathrm{~mm}$. In this case, the main optical principle lies in the variation in refractive index within the sample that, in turn, gives rise to an increase in direct backscattering of incoming photons ${ }^{15}$. The depth resolved structural data $I(t)$ are obtained directly by inverse Fourier transforming the detected frequency spectrum given $b^{14}$ :

$$
I(t)=F T^{-1}\left\{\frac{1}{4}|s(\omega)|^{2}\left[\left(\int_{-\infty}^{\infty} r(\omega, z) e^{\left(\frac{i 2 n(\omega, z) \omega z}{c}\right)} d z\right)+1\right]^{2}\right\}
$$

where $s(\omega)$ is the source field amplitude spectrum, $r(\omega, z)$ is the backscattering coefficient resulting from the structural features of the sample, and $F T^{l}$ is the inverse Fourier transform operator.

FD-OCT measurement and analysis has also been applied to the depth characterization of PNCs ${ }^{16}$. The measurement beam of the OCT microscope penetrates the sample at a specific point and this intensity variation is called an A-scan; the microscope then continues the scanning along a straight line and the resulting combined A-scan series is called the Bscan. The probing beam then raster scans an area of the sample thus producing a two-dimensional B-scan representation of the sample with a spatial resolution of $4 \mu \mathrm{m}$. By using special image processing software (ImageJ) it is possible to reorder the B-scans in order to produce an en-face (depth) representation of the sample as a sequence of images called Cscans. Every individual C-scan represents a 2-D depth area that reveals the internal structure of the material at that particular depth.

It is worth noting that the resulting signal-to-noise ratio of the measurements decreases as the penetration depth increases; however, for the materials investigated in this work the probing depth was up to $2 \mathrm{~mm}$. An additional issue that may cause erroneous data or possible saturation of the optical photodetector arises from reflections near the surface of the sample. To avoid this, a thin layer of refractive index matching liquid (aliphatic and alicyclic hydrocarbons, hydrogenated terphenyl, refractive index value of $1.570 \pm 0.0002)$ was applied on the surface of the sample. In addition, the first few depth layers were not used for the analysis of the samples. Figure 1 shows two representative C-scans (depth is approximately $100 \mu \mathrm{m}$ ) from a PNC with clay platelets $4 \%$ loading and poor dispersion (a) and silica beads $1 \%$ loading and good dispersion (b).

The images from the FD-OCT measurements provide a direct visual representation of the samples under investigation, with a typical measurement area being approximately $4 \mathrm{~mm}^{2}$. However, it would be favorable to actually provide numerical values associated to the level of dispersion. For this reason, it was necessary to apply image processing tools for the analysis.

The process started by choosing a layer that is not close to the surface of the sample, but is, upon inspection, representative of the PNC. The image was then split into its horizontal, vertical and diagonal wavelets of the same order; this was done in order to enhance the spatial information of the selected C-scan. Each wavelet image was subsequently divided into a square $16 \times 16$ segment array (256 segments in total). For each segment, the average intensity was first calculated, followed by the standard deviation of each segment pixel against the average intensity. This standard deviation value was then assigned to that particular segment. This process was performed for all 256 segments and then the average of all 256 standard deviations was computed. This value was then assigned to that particular C-scan representing the material for the specific wavelet analysed. This process was performed for all three wavelet images and the three standard deviations were averaged into one value that was then assigned to the specific material under characterization.

In order to provide comparative data, density measurements were performed for all materials studied. Table 1 shows the results from the density and OCT image analsysis of the epoxy resin PNC embedded with silica beads. 


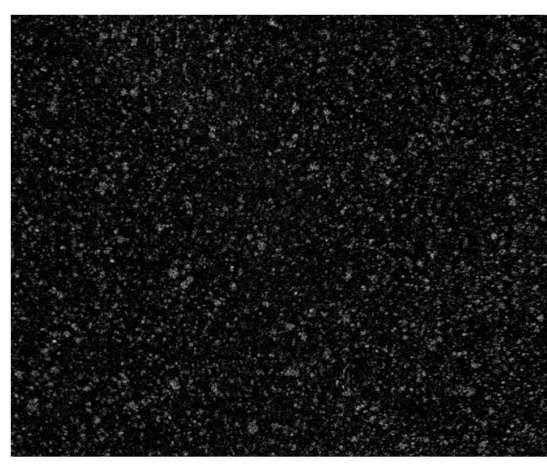

(a)

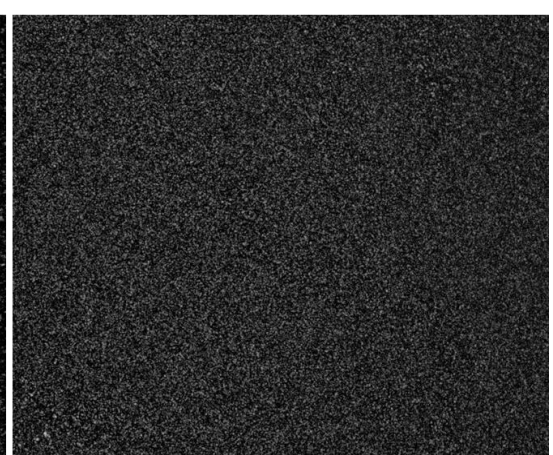

(b)

Figure 1: Optical coherence tomography individual C-scans for two PNCs: (a) 4\% clay loading with poor dispersion and (b) $1 \%$ silica beads with good dispersion

\begin{tabular}{|c|c|c|}
\hline $\begin{array}{c}\text { Particle Loading } \\
\text { (wt.\%) }\end{array}$ & $\begin{array}{c}\text { Density } \\
\text { (g/cm }^{\mathbf{3}} \text { ) }\end{array}$ & $\begin{array}{c}\text { Standard deviation } \\
\text { (average) }\end{array}$ \\
\hline $0.1(0.102)$ & 1.1941 & $21.89 \pm 1.35$ \\
\hline $0.2(0.200)$ & 1.1947 & $22.87 \pm 1.12$ \\
\hline $0.5(0.505)$ & 1.1959 & $23.55 \pm 0.93$ \\
\hline $1.0(0.994)$ & 1.1987 & $26.02 \pm 0.83$ \\
\hline
\end{tabular}

Table 1: Density measurements compared to FD-OCT measurements (average standard deviation) for the PNC samples with silica beads

For the case of the silica PNCs, the density measurements show a distinction with increasing loading and this is also the case for the FD-OCT measurements. It can also be seen that the standard deviation increases as the particle loading increases and this is due to higher concentration thus producing higher optical scatter; in addition, the tolerances provided reduce as the level of loading increases. It is quite interesting to notice the absolute trend between the density and OCT data are very similar indeed. The close agreement between the two methods could therefore be attributed to the good level of dispersion achieved for these specific PNCs. The clear distinction between different levels of loading is also facilitated by the fact that the silica beads themselves are very well defined in terms of physical characteristics and dimensions as shown in figure $2(a)$.

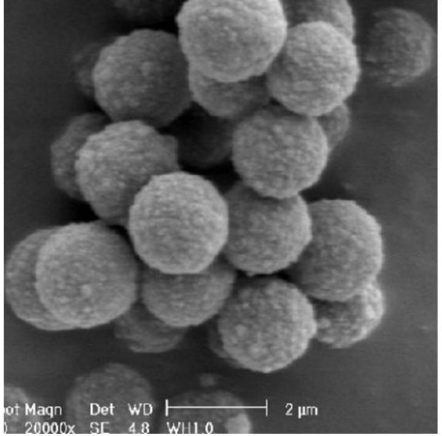

(a)

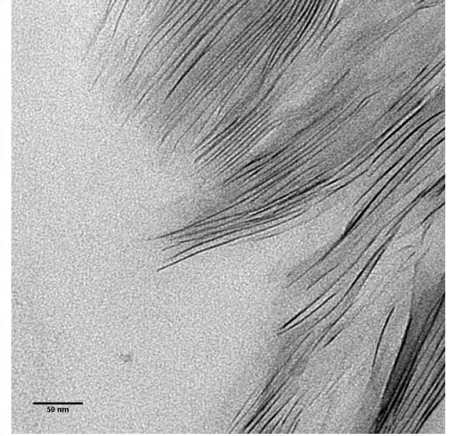

(b)

Figure 2: TEM images showing the physical characteristics of silica beads (a) and clay platelets (b); the scale bars are 2 $\underline{\mu \mathrm{m} \text { and } 50 \mathrm{~nm} \text { respectively. }}$ 
However, the situation changes for the PNCs embedded with clay platelets. Such nanofillers are shown in figure 2(b); it is evident that their physical characteristics are quite different and their most dominant feature is the high aspect ratio (length over width). This can have a quite important effect on the optical backscatter during the OCT measurements.

Table 2 shows the results from the PNC samples embedded with clay platelets. Starting with the density measurements, there seems to be a very small difference within the $1.0 \%$ loading regime; the density should increase not only as the content does, but also as the dispersion improves but it is not possible to discriminate between levels of dispersions at low particle levels. At higher concentrations (4\%), density measurements can reveal a small discrimination between levels of homogeneity but the difference appears to be at the third decimal place.

The OCT measurements show a clearer picture on the other hand. For the low particle loading case, there seems to be a distinctive difference between the dispersion profiles in terms of the calculated standard deviation. Also, the stated tolerance decreases as the dispersion improves and this due to the fact the optical scatter becomes more uniform. For the $4 \%$ loading, the difference between poor and good dispersion is even more profound in terms of value deviation; similar to before, the tolerance reduces as the homogeneity increases but a lower rate compared to the $1 \%$; this is due to the high particle content.

\begin{tabular}{|c|c|c|}
\hline $\begin{array}{c}\text { Particle Loading } \\
\text { (wt.\%) }\end{array}$ & $\begin{array}{c}\text { Density } \\
\left(\mathbf{g} / \mathbf{c m}^{\mathbf{3}}\right)\end{array}$ & $\begin{array}{c}\text { Standard deviation } \\
\text { (average) }\end{array}$ \\
\hline$\underline{1.0}$ & & \\
Poor Dispersion & 1.1406 & $14.80 \pm 2.61$ \\
Moderate Dispersion & 1.1416 & $18.89 \pm 2.03$ \\
Good Dispersion & 1.1398 & $19.32 \pm 1.60$ \\
\hline$\underline{2.0}$ & & \\
Good Dispersion & 1.1448 & $18.96 \pm 1.06$ \\
\hline$\underline{4.0}$ & & \\
Poor Dispersion & 1.1564 & $18.55 \pm 1.44$ \\
Good Dispersion & 1.1582 & $23.29 \pm 1.11$ \\
\hline
\end{tabular}

Table 2: Density measurements compared to FD-OCT measurements (average standard deviation) for the PNC samples $\underline{\text { with clay platelets }}$

\section{FRAUNHOFER WAVEFRONT CORRELATION}

Pattern recognition has been an extensively researched area over the course of decades; its origin has been attributed to the original work of Vander Lugt $^{17}$ and Weaver and Goodman ${ }^{18}$ producing two separate classes of optical (and subsequently hybrid digital/optical) configurations, termed as $4 f$ and joint-transform correlators respectively.

In this case, the primary aim of the research has always been the accurate recognition and tracking and even reconstruction of two and three-dimensional (2D and 3D respectively) objects and targets. This is achieved by comparing an image containing the object to be identified (reference) with an image containing an unknown target (input) via the convolution operation based in the spatial frequency domain, an operation commonly referred to as the correlation theorem (derived from its convolution counter-part). A wide variety of such generic or custom-designed filters have been mathematically formulated, simulated and experimentally verified each capable of achieving specific levels of invariance relating to rotation ${ }^{19-21}$, $\mathrm{scale}^{22,23}$, and non-Gaussian (additive, multiplicative and disjoint) noise ${ }^{24}$, with fully complex and phase-quantised spectral information ${ }^{25}$. In the case of PNCs, spatial filtering techniques can also provide very useful tools for the analysis and subsequent characterisation of various dispersion profiles ${ }^{15}$.

A propagating 2-D optical wavefront can be defined, in Cartesian spatial terms, as a function $f(x, y)$, where its value at a specific point is defined as the intensity of the wavefront. If this pattern is transmitted through a neat resin polymer, it can be regarded as the reference function. However, if the very same wavefront propagates through a PNC, it will become spatially modulated (in other words, diffused) depending on the combination of its particle loading content and dispersion characteristics; this function can be regarded as the input function $g(x, y)$. It comes natural that different PNCs will exhibit different input functions and if they are correlated against the reference, dispersion properties may be attempted to be analysed. 
The setup for this analysis, consisted of a He:Ne laser source, with a wavelength of $633 \mathrm{~nm}$ and an optical power of 1 $\mathrm{mW}$. The beam was directed via mirrors onto a diffraction grating that generated a propagated 2-D pattern of dots. Using a subsequent aperture, a section of the wavefront was allowed to transmit through the material under investigation that was placed at an angle of $90^{\circ}$ relative to the axis of light propagation. A CCD camera placed at an angle relative to the axis of optical propagation (to avoid capturing the zero order from the diffraction grating) captured the emerging patterns from the samples. Figure 3 shows the experimental setup of the wavefront correlation system.

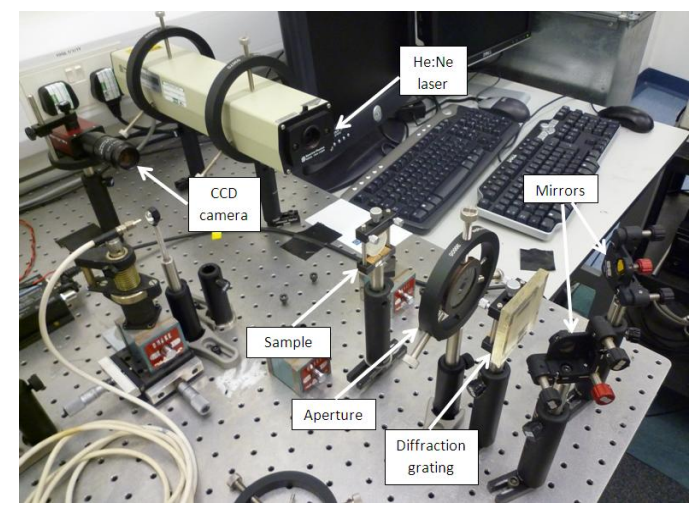

Figure 3: The optical system for the wavefront correlation characterization technique

The optical wavefront propagates through the sample itself and the resulting diffused wavefront is captured by the CCD camera. Specific properties of the nanofillers play a significant contribution to the overall optical diffusion mechanism, in addition to the coherence of the emerging wavefront; average particle size, refractive index, particle loading, dispersion level, in addition to thickness of the sample. These effects can be seen in figure 4, showing spatially modulated patterns resulting from neat resin and embedded with two different types of nanofillers.

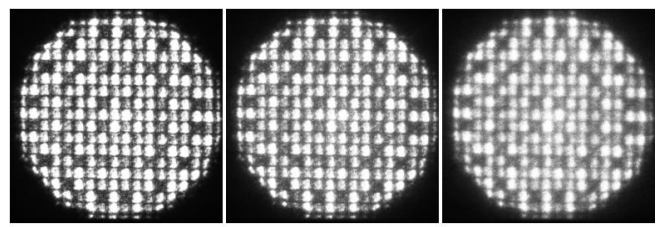

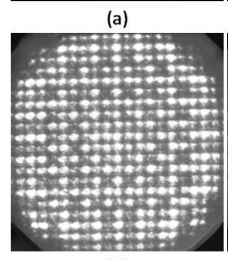

(d) (b)

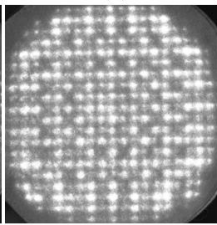
(c)

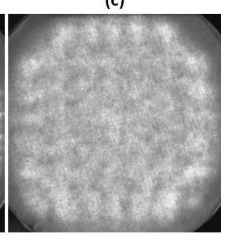

(f)

Figure 4: Spatially modulated wavefront patterns resulting from neat epoxy resin (a), $2 \%$ clay loading with good dispersion (b), $4 \%$ clay loading with poor dispersion (c), neat epoxy resin (d), $0.1 \%$ silica loading (e) and $0.5 \%$ silica loading (f)

The emerging pattern from the diffraction grating itself expanded as it propagated through space, but its dimension, when incident on the samples under investigation, was $20 \mathrm{~mm}$ in diameter. The resolution of the camera was $980 \mathrm{x} 1000$ pixels, meaning that the spatial resolution of each pixel was $18 \times 18 \mu \mathrm{m}^{2}$.

In terms of signal processing, two of the main advantages that the correlation theorem offers, is computational efficiency and the opportunity to manipulate the spectra of the reference and input function prior to the required complex 
multiplication and transformation to the correlation plane via the inverse Fourier transform operation. For a given set of 2-D functions (reference and input), their Fourier transforms (spectra) are first calculated. In this case, lower spatial frequencies are located at the centre of the Fourier plane, while higher spatial frequencies extend outwards. It is then possible to apply a window to block certain frequencies and to allow all the remaining to be used for the subsequent stages of the analysis (complex multiplication and inverse Fourier transform). For the analysis of the PNCs, the iteration algorithm applies an iterative low spatial filter; that is for each step, the algorithm blocks the higher spatial frequencies in steps; $0 \%$ (no filtering) to $90 \%$ (wide low pass filtering) in steps of 10 . For each step, the signal-to-noise (SNR) ratio of the resulting correlation plane is calculated. In this case, the SNR is defined as the energy ratio of the central region (signal) of the correlation plane, divided by the remaining plane (noise). Therefore, for each set of 2-D functions, a total of 10 SNR values were obtained, that were subsequently averaged to produce a characterization value for the particular input function (specific PNC) analysed.

In order to have a reference value that can be used for normalization purposes, the above iteration process was also repeated on the auto-correlation function (reference, i.e. optical wavefront from neat epoxy). It was then possible to normalize the SNR values from all the investigated samples against that of the neat epoxy resin. Figure 5 shows the cross-correlation curves produced by the two different classes of materials (clay and silica).

For the clay enhanced PNCs, there seems to be a very clear distinction between levels of loading and dispersion profiles. As expected, the samples closest to the auto-correlation value correspond to those with lower particle content (loading); as the dispersion profile becomes more inhomogeneous, the SNR decreases. As the level of loading increases, the resulting SNR decreases accordingly. According to these results, it seems that there is little difference between moderate and poor dispersion levels, even though poor exhibits the lowest SNR in the case of $1 \%$ loading. The silica bead enhanced PNCs, exhibit a fairly similar trend - decreasing SNR with increasing content. Again, it is easy to discriminate between different loading levels. From a relative perspective between the clay and silica samples, the SNR values are different, but this is due to the physical characteristics of the nanofiller themselves - geometrical morphology and refractive index being the most dominant parameters.
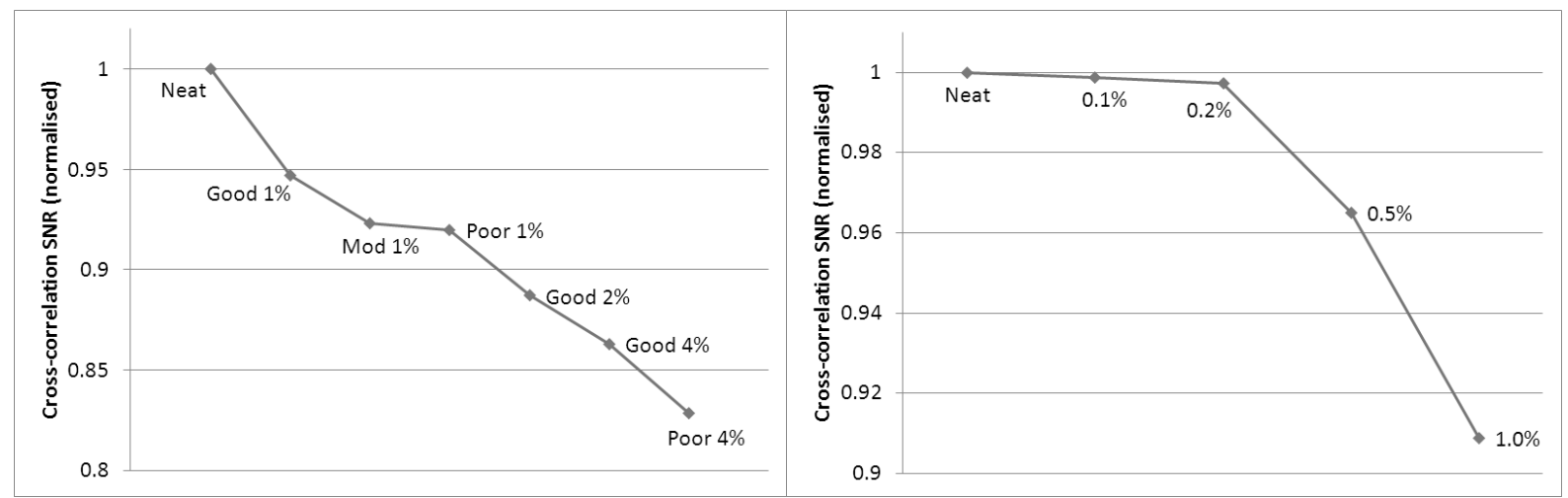

Figure 5: Cross-correlation normalized SNR plots of varying loading and dispersion for the PNC clay (left) and silica (right) samples resulting from the FWC analysis

\section{OSCILLATORY PHOTON CORRELATION SPECTROSCOPY}

Photon correlation spectroscopy (PCS) is a powerful light scattering technique that has been investigated in great detail over the course of the last few decades. It was initially used primarily to study specific properties of living cells ${ }^{26,27}$ and other biological systems ${ }^{28}$. The work was then subsequently expanded into the use of more coherent light sources thus enabling the formulation and use of the photocurrent autocorrelation function for scattered light intensity ${ }^{29}$ to enable the more accurate analysis of specific properties of small scattering volumes.

Subsequent work concentrated on the determination of the size of particles undergoing Brownian motion ${ }^{30-32}$ present in liquids. Temporal resolution has also been improved by Fourier-based photon correlation ${ }^{33}$ and more recently there has been a return to the use of incoherent light as the main source for PCS experiments ${ }^{34}$. PCS analysis relies on the 
Brownian motion of suspended particles in a solution form (liquid). In this case, the Siegert relationship ${ }^{35}$ yields the required autocorrelation function (ACF) that enables the determination of specific measurands of the solution under investigation.

The experimental setup of photon spectroscopy and the subsequent analysis can also be modified to accommodate the study of solid materials ${ }^{15,36}$. The main idea in this case, is to cross two coherent laser beams at a specific point in space, such that they form an ellipsoid volume with interference fringes. Though the materials under investigation were solid, they can be regarded as static media; however, by oscillating them through the formed interference volume, the process becomes dynamic; the interaction of the nanofillers within the PNC matrix with the optical fringes produces scattered photons that can be collected, analysed and through the analysis of the ACF, specific properties of the materials under investigation can be revealed.

Figure 6 shows the experimental schematic for the oscillatory PCS (Os-PCS) measurements. The main laser source was a He:Ne cavity laser, with a wavelength of $633 \mathrm{~nm}$, power of $10 \mathrm{~mW}$ and mounted in such a way that the exit light was vertically polarised. The subsequent $\lambda / 2$ waveplate was used as a power control, in order to adjust the intensity of the laser beams. A non-polarising beam-splitter produced two identical (from a coherence and polarization perspective) beams of equal intensity. Two mirrors were used in order to cross the beams at a point in space; the half angle of intersection was $5^{\circ}$, the fringe spacing was $3.6 \mu \mathrm{m}$, the number of fringes was 276 , while the dimensions of the ellipsoid were $1 \times 11.5 \times 1 \mathrm{~mm}^{3}$. The materials under investigation were mechanically oscillated (using a suitable piston transducer and function generator) through the ellipsoid volume. The back-scattered radiation was collected using a short focal length lens which coupled the light to a single-mode optical fibre. The output of the fibre was coupled on a single photon counter producing equal intensity pulses, each corresponding to a single photon event. The sequences were analysed by a suitable correlator board and the software controlling it produced the experimental ACFs.

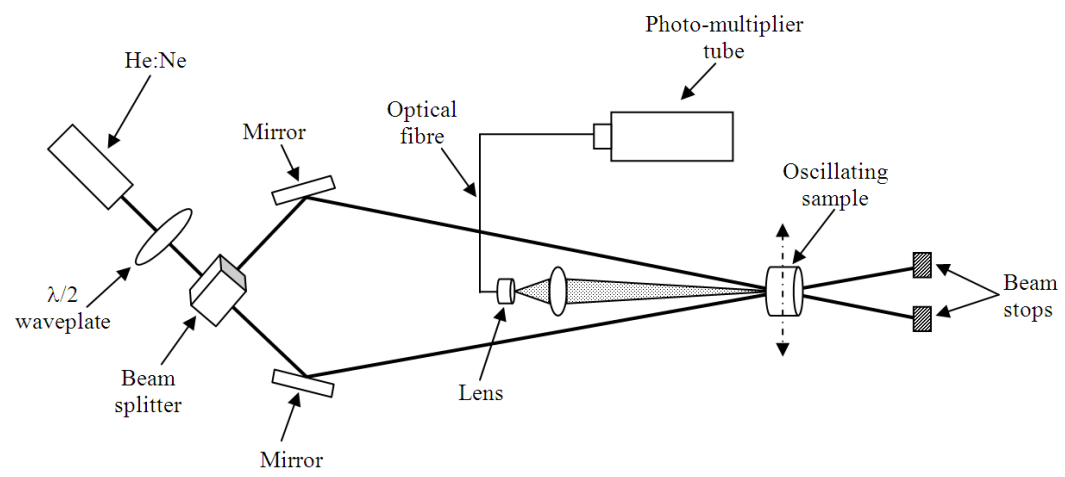

Figure 6: The experimental setup of the oscillatory photon correlation spectroscopy measurements

Though the ACF is, as mentioned, based on the Siegert formula, its theoretical form resulting from a sinusoidal flow has also been formulated ${ }^{37}$ and simplified ${ }^{38}$ to the following relationship:

$$
R(\tau)=\frac{k^{2} E\left[K_{p}^{2}\right] g_{0} \sqrt{\pi}}{8 \beta} \exp \left(-2 u_{m}^{\prime 2} \beta^{2}\right)\left(1+J_{0}\left(D u_{m}^{\prime}\right)\right)
$$

where $k$ is a constant associated with the optical power and detector sensitivity, $\beta$ is related to the half angle of the intersection of the laser beams and the beam waist, $u_{m}^{\prime}$ is related to the velocity and frequency of the sinusoidal oscillation, $J_{0}$ represents the Bessel function operator and $D$ is related to the half angle of the intersection of the laser beams and the wavelength of the light source. The two most important parameters in this case are $K_{p}$, which characterizes the particle scattering cross section (physical characteristics related to the dispersion profile) and $g_{0}$ that is 
related to the average number of particles per unit length in the fringe volume (particle loading). The combination of different levels of particle loading and dispersion therefore directly affect the dynamic range of the experimentally obtained ACFs.

For this experimental study, the two classes of materials (clay and silica embedded PNCs) were placed at the centre of the ellipsoid region and oscillated using a sinusoidal excitation such that the effective scanning area was approximately 3 $\mathrm{mm}$. The axis of oscillation was perpendicular to the bisector of the angle produced by the two interfering laser beams. For each material, three different locations of each material were analysed and the experimental ACFs were averaged for each case.

In order to facilitate the discrimination capability between different loadings and dispersion profile, the logarithmic trendline of the ACFs were computed and plotted as a function of time lag given by the correlator board. Figure 7 shows the plotted trendlines obtained for all the materials investigated in this study.

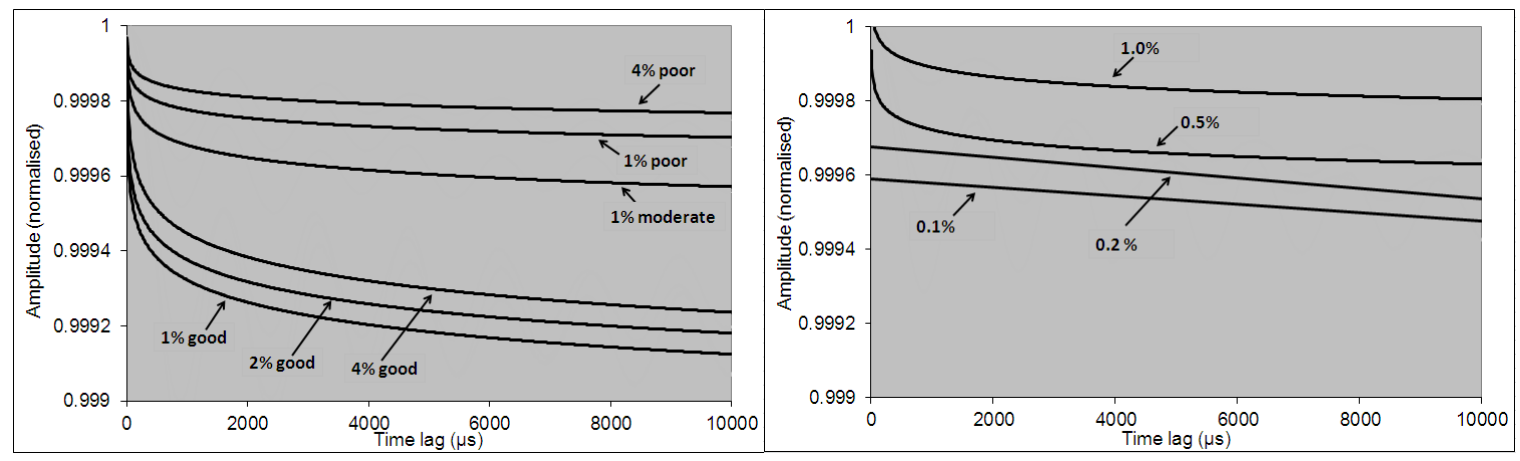

Figure 7: Experimental ACFs obtained for the clay enhanced (left) and silica enhanced (right) PNCs

Starting with the clay enhanced PNCs, the materials exhibiting the highest dynamic responses are those of good dispersion. More interestingly there is a clear step change for loading of $1 \%, 2 \%$ and $4 \%$. The slightest variation in the dispersion profile has a significant effect on the dynamic range; for the $1 \%$ particle loading case, poor and moderate levels of dispersion are considerable higher in value compared to the good level of dispersion, while in a comparative sense, they are both numerically close to each other and still discriminable. The lowest dynamic range response was, quite evidently, produced by the $4 \%$ poorly dispersed PNC.

For the silica PNCs, the dispersion characteristics were the same for all samples and the difference was only in the level of loading. Again, it may be seen that discrimination is easy between all materials. It is interesting to note that by comparing the two classes of PNCs, the normalized amplitudes appear to be an order of magnitude out with respect to each other (by comparing the $1 \%$ clay and $1 \%$ silica samples). This is due to the different scattering properties of the nanofillers (size, clustering effects, physical geometry, aspect ratio, refractive index).

\section{CONCLUSIONS}

This work presented the application of three different approaches to the characterization of PNC materials. Compared to existing conventional and standard methods (such as TEM and XRD), the reported optical techniques can differentiate between different classes of materials based on their level of loading and dispersion profiles from small to larger scales. Most importantly, they are non-destructive or invasive and can easily be adapted into in-situ systems for rapid characterization of components in industrial situations.

Fourier-domain optical coherence tomography provides a relatively rapid measurement accurate approach. The technique works in the infra-red part of the spectrum and relies of refractive index boundaries inside the material under investigation. The resulting back scattered radiation is analysed and by suitable deconvolution, spatial characteristics of the material for different depths are revealed. This technique provides a visual representation of the inside structure and 
by segmentation, wavelet decomposition and correlation plane estimation, the homogeneity of the material can be studied.

Fraunhofer wavefront correlation is a transmission optical technique that can analyse much larger sections of materials in reduced time. In this case, an optical wavefront passes through the material and becomes spatially modulated due to the presence of nanofillers. Through Fourier-domain iterative low pass spatial filtering, the correlation plane is computed and the resulting signal-to-noise ratio gives a quantitative metric compared to the auto-correlation function. This technique can also discriminate between loading levels and dispersion profiles.

Finally, oscillatory photon correlation spectroscopy can also yield specific responses of materials depending on their physical structural characteristics. In this case, the material under investigation is mechanically oscillated through an ellipsoid volume full of interference fringes. The optical interaction of the nanofiller with the fringes produce scattered photons that are collected and analysed. The auto-correlation analysis of captured photon sequences can provide a useful characterization tool between different materials.

The control of the dispersion properties in PNCs is one of the most important parameters currently affecting (and halting in many cases) the commercialization of such polymeric structures. The optical techniques reported, offer rapid and accurate characterization platforms that can be applied in academic and industrial environments that may enable researchers to better understand the mixing processes required to achieve repeatable dispersion profiles for a wide range of multiphase systems.

\section{ACKNOWLEDGEMENTS}

This work, funded by the United Kingdom Department for Business, Innovation and Skills (National Measurement Office) is part of the Materials Programme 2010-2013. The authors would also like to thank Dr Amir Rezai (BAE Systems (Operations) Ltd), Dr Tim Young and Mr Peter Wolliams for their technical support and advice. C Queen's Copyright Printer and Controller of HMSO, 2012.

\section{REFERENCES}

[1] Broughton, W.R., [Characterization of nanosized filled particles in polymeric systems: a review], National Physical Laboratory, ISSN:1754-2979, (2008).

[2] Wagenere, R. and Reisigner, T.J.G., "A rheological method to compare the degree of exfoliation of nanocomposites", Polymer, 44, pp. 4573-4588, (2003).

[3] Zhao, J., Morgan, A.B. and Harris, J.D., "Rheological characterization of polystyrene-clay nanocomposites to compare the degree of exfoliation and dispersion", Polymer, 46, pp. 8641-8660, (2005).

[4] Decker, C., Keller, L., Zahouily and K., Benfarhi, S., "Synthesis of nanocomposite polymers by UV-radiation curing", Polymer, Volume 46, pp. 6640-6648 (2005).

[5] Mai, Y.-W., Yu and Z.-Z., [Clay-Acrylate Nanocomposite Photopolymers], Chapter 7, Woodhead Publishing Limited, Oxford, United Kingdom (2006).

[6] Utracki, L.A., [Clay-Containing Polymeric Nanocomposites], Rapra Technology Limited, (2004).

[7] Wang, Z. L., "New developments in transmission electron microscopy for nanotechnology", Advanced Materials, 15, pp. 1497-1514, (2003).

[8] Vermogen, A., Masenelli-Varlot, K., Seguela, R., Duchet-Rumeau, J., Boucard, S. and Prele, P., "Evaluation of the structure and dispersion in polymer-layered silicate nanocomposites" Macromolecules, 38, pp. 9661-9669, (2005).

[9] Morgan, A. B. and Gilman, J. W., "Characterization of polymer-layered silicate (clay) nanocomposites by transmission electron microscopy and X-ray diffraction: a comparative study", Journal of Applied Polymer Science, 87, pp. 1329-1338, (2003).

[10] Schmitt, J.M. "Optical coherence tomography (OCT): a review", IEEE Journal of Selected Topics in Quantum Electronics, Volume 5, pp. 1205-1215 (1999).

[11] Podoleanu, A.G., "Optical coherence tomography", The British Journal of Radiology, 78, pp. 976-988, (2005).

[12] Leitgeb, R., Hitzenberger, C. K. and Fercher, A. F., "Performance of Fourier domain vs. time domain optical coherence tomography", Optics Express, 11 (8), pp. 889-894, (2003).

[13] Choma, M. A., Sarunic, M. V., Yang, C. and Izatt, J. A., "Sensitivity advantage of swept source and Fourier domain domain optical coherence tomography", Optics Express, 11 (18), pp. 2183-2189, (2003). 
[14] Tomlins, P.H. and Wang, R.K., "Theory, developments and applications of optical coherence tomography", Journal of Physics D: Applied Physics, 38, pp. 2519-2535, (2005).

[15] Koukoulas, T., Broughton, W.R., Tedaldi, M. and Theobald, P.D., "Novel non-contact optical characterization methods of polymeric nanocomposite structures based on their particle loading and dispersion profile", Proc. SPIE 8082, Optical Measurement Systems for Industrial Inspection VII, 808220:1-808220:11, (2011).

[16] Williams, J., Broughton, W., Koukoulas, T. and Rahatekar, S.S., "Plasma treatment as a method for functionalising and improving dispersion of carbon nanotubes in epoxy resins", Journal of Materials Science, DOI: 10.1007/s10853012-6830-3, (2012).

[17] Vander Lugt, A., "Signal detection by complex spatial filtering”, IEEE Transactions on Information Theory, pp.139$145,10,(1963)$.

[18] Weaver, C.S. and Goodman, J.W., “A technique for optically convolving two functions”, Applied Optics, 5 (7), pp. 1248-1249, (1966).

[19] Lefebvre, D., Arsenault, H.H. and Roy, S., "Nonlinear filter for pattern recognition invariant to illumination and to out-of-plane rotations", Applied Optics, 42 (23), pp. 4658-4662, (2003).

[20] XBermudez, J.G., "Object recognition using cylindrical harmonic filter”, Optics Express, 12(13) pp. 3046-3049, (2004).

[21] Munshi, S., Beri, V.K. and Gupta, A.K., "Hybrid digital-optical correlation employing a chirp-encoded simulatedannealing-based rotation-invariant and distortion-tolerant filter", Applied Optics, 46 (20), pp. 4304-4319, (2007).

[22] Xli., Y and Rosen, J., "Scale-invariant recognition of three-dimensional objects by use of a quasi-correlator", Applied Optics, 42(5), pp. 811-819, (2003).

[23] Yin, Q., Shen, L., Kim, J.-N. and Jeong, Y.-J., "Scale-invariant pattern recognition using a combined Mellin radial harmonic function and the bidimensional empirical mode decomposition", Optics Express, 17 (19), pp. 16581$16589,(2009)$.

[24] Towghi, N. and Javidi, B., "Image recognition in the presence of non-Gaussian noise with unknown statistics", Journal of the Optical Society of America A., 18 (11), pp. 2744-2753, (2001).

[25]T. Koukoulas, R.C.D. Young and C.R. Chatwin, "Quantised phase filters with binary low and high pass amplitude response: the effect of quantisation for scale and rotation input distortions", Lasers and Optics in Engineering, 43(9), pp. 963-976, (2005).

[26] Carlson, F.D., "The application of intensity fluctuation spectroscopy to molecular biology", Annual Review of Biophysics and Bioengineering, 4, pp. 243-264, (1975).

[27] Mustacich, R.V. and Ware, B. R., "Observation of protoplasmic streaming by laser-light scattering", 33(11), pp. 617-620, (1974)

[28] Maeda, T and Fujime, S., "Quasielastic light scattering under optical microscope", Review of Scientific Instruments, 43, pp. 566-567, (1972).

[29] Herbert, J.T. and Acton, J.D., "Photon correlation spectroscopy of light scattered from microscopic regions", Applied Optics, 18 (5), pp. 588-589, (1979).

[30] Chowdhury, D.P., Sorensen, C.M., Taylor, T.W., Merklin, J.F. and Lester, T.W., “Application of photon correlation spectroscopy to flowing Brownian motion systems", Applied Optics, 23 (22), pp. 4149-4154, (1984).

[31] Bronk, B.V. and Smith, M.J., "Photon-correlation spectroscopy for small spherical inclusions in a micrometer-sized electrodynamically levitated droplet", Optics Letters, 18 (2), pp. 93-95, (1993).

[32] Mao, S., Shen, J., Thomas, J.C., Zhu, X., Liu, W. and Sun, X., "Improved inversion procedure for particle size distribution determination by photon correlation spectroscopy", Applied Optics, 51 (25), pp. 6220-6226, (2012).

[33] Brokman, X., Bawendi, M., Coolen, L. and Hermier, J.-P., "Photon-correlation Fourier spectroscopy", Optics Express, 14 (13), pp. 6333-6341, (2006).

[34] Salerno, D., Brogioli, D., Ziano, R. and Mantegazza, F., "Photon correlation spectroscopy with incoherent light", Optics Express, 19 (27), pp. 26416-26422, (2011).

[35] Berne, B.J. and Pecora, R., [Dynamic Light Scattering], Wiley, (1976).

[36] Koukoulas, T., Broughton, W.R., Theobald, P.D. and Tedaldi, M., "Characterisation of solid complex multiphase systems based on oscillatory photon correlation spectroscopy", Optics Letters, 35 (22), pp. 3754-3756, (2010).

[37] Durrani, T.S. and Greated, C.A., [Laser systems in Flow Measurement], Plenum Press, (1977).

[38] Sharpe, J. P. and Greated, C. A., "A Stochastic Model for Photon Correlation Measurements in Sound Fields", Journal of Physics D: Applied Physics, 22, pp. 1429-1433, (1989). 
2013-01-25

\section{Optical characterisation of polymeric nanocomposites using tomographic,} spectroscopic and Fraunhofer wavefront assessme

\section{Koukoulas, Triantafillos}

SPIE - International Society for Optics and Photonics

Triantafillos Koukoulas, William R. Broughton, John Williams and Sameer Rahatekar. Optical characterisation of polymeric nanocomposites using tomographic, spectroscopic and Fraunhofer wavefront assessment.

http://dx.doi.org/10.1117/12.981182

Downloaded from Cranfield Library Services E-Repository 